\title{
Bayesian update of the residual life of a concrete structure using inspection data
}

\author{
Gaowei Xu, Viliam Makis \\ Department of Mechanical \& Industrial Engineering, University of Toronto \\ 27 King's College Circle, Toronto, Ontario M5S 1A1 Canada \\ Gaowei.xu@mail.utoronto.ca; Makis@mie.utoronto.ca
}

\begin{abstract}
This article presents a model of a deteriorating concrete structure subject to external load and inspection. The structure naturally deteriorates in time due to the environmental effects and the changes in the construction materials. The deterioration process is modeled as a gamma process. The stochastic process of load arrivals is generated by a Poisson process and the variability of the random loads is assumed to be governed by a generalized Pareto distribution. Upon inspection, the parameters of the gamma distribution are updated using the Bayesian method. Thus, after each inspection, the resistance of the concrete structure is modified and the corresponding lifetime distribution of the structure can be estimated. An example of concrete girders is provided to illustrate the whole methodology.
\end{abstract}

Keywords: Concrete structure, Gamma process, Pareto distribution, Bayesian updating, Residual life.

\section{Introduction}

During the lifetime of a civil engineering structure, the resistance of the structure will decrease due to the influence of environmental conditions, external loads, and construction materials degradation. For concrete structures in the winter of high-latitude regions, spreading de-icing salt on concrete will allow chloride to penetrate into the concrete and corrode the steel bars embedded within. The daily spreading of de-icing salt can also make a freezing-thaw cycle that accelerates the cracking of concrete. Many hydraulic concrete structures have a similar problem such as the sea water penetrating into the concrete port. Highway bridges are also subjected to the impacts from heavy haul vehicles, which are another main factor affecting the structure's reliability. These heavy loads can gradually damage the structure and intensify concrete cracking, which allows chloride to corrode the reinforcement bars faster. In common structural design, a structure fails when the load exerting on the structure exceeds its bearing capacity. As the structure ages, the probability of failure will increase.

A typical deterioration of civil engineering structure can be expressed as a monotonically decreasing process, as the corrosion process and the growth of concrete crack width are irreversible if there is no repair work implemented. Enright [1] adopted a parabolic function, $g(t)=1-k_{1} t+k_{2} t^{2}$, as the resistance degradation function that referred to the fraction of initial resistance of a structural member remaining at time $t$. Suo [2] directly used some physical and mechanical models to simulate the corrosion process of reinforcement bars in reinforcement concrete (RC) structure. The proportion of a concrete surface with crack widths exceeding the limit width at time $t, d_{\text {crack }}(t)$, was the main index investigated.

Except for concrete structure, there are also many studies on other monotonically deteriorating civil engineering structures. Miran et al. [3] investigated the reliability of buried pipelines with external defects. They assumed the initiation time of each defect was considered to follow a gamma distribution, and the defect with a larger detected dimension occurred earlier. Hong [4] established the way to calculate the serviceability, $p_{t}$, of an asphalt road. Traffic volume, material's deterioration, and a normally distributed error term were involved, and each parameter related to these factors was assumed to be normally distributed.

Traffic load on a bridge is classified by axle load. From the typical diagram of axle loads, heavier axle loads occur fewer times, so the distribution of axle loads is close to a negative exponential distribution. In the paper by Noortwijk et al. [5], the authors assumed that only the loads exceeding a critical level $l_{0}$ can affect the reliability, and they applied generalized Pareto distribution to describe the loads. The paper gives a dike section of Den Helder sea defense affected by the wave run-up level as an example. The periodic external loads exceeding a certain sea level $l_{0}$ were defined as the external loads that might affect the sea defense. The stochastic process of loads was defined by a homogeneous Poisson process. 
In order to predict the performance of a civil engineering structure, the initial distribution will be estimated based on the past experience and historical data. However, when new inspection data becomes available, the initial distribution should be updated by using the new data. As the deteriorating scenario and the deterioration rate can be different due to the variability of the surrounding environment, the initial assumption would not be precise anymore. Bayesian updating is a useful method to update the initial distribution of the process parameters. Unlike maximum likelihood estimation method (MLE), Bayesian updating does not require a large number of samples to estimate the parameters, which is an ideal method to involve the inspecting data [6]. As this method is complemented by recalculating the related parameters of the initial distribution of the deterioration process, some researchers use the term Bayesian parameter estimation method [7].

The paper is organized as follows. A gamma process is selected to model the deterioration of the reinforcement concrete (RC) bridge structures, as gamma process is widely used for modeling wear processes [8]. The periodic traffic loads that exceed a certain value occur randomly according to a Poisson process, and the loads' exceedance over the certain critical value is governed by a generalized Pareto distribution. The objective is to obtain the distribution of structural lifetime, which is a function of deterioration process and random loads. Finally, the Bayesian parameter estimation method is used to update the distribution of the process parameters using inspection data and to calculate the updated residual life of the structure.

\section{Random process of a deteriorating concrete structure}

Since the deterioration of concrete structures is a complex process that involves many factors, to simplify the model, the following assumptions should be added.

(1) For RC bridge structures, their deterioration is observable and it is measured by the degrading rate of the flexural capacity of the girders. The corrosion of reinforcement bars begins when the bridge begins to operate.

(2) The impact of natural disasters (such as earthquakes) is not considered.

(3) The loads are regarded as a series of pulses so that their duration time can be neglected.

The random variable $X(t)$ representing the total deterioration of structural resistance in $t$ time units is assumed to have a gamma distribution, with the probability density function given by

$$
g(x \mid \alpha(t), \beta)=\frac{\beta^{\alpha(t)}}{\Gamma(\alpha(t))} x^{\alpha(t)-1} e^{-\beta x}
$$

where $\alpha(t)$ is the shape parameter which is the function of time, and $\beta$ is the scale parameter. The gamma process has the following properties:

$\cdot \mathrm{X}(0)=0$ with probability one;

- The increment between time $t_{1}$ and any time $t_{2}$ after $t_{l}, X\left(t_{2}\right)-X\left(t_{1}\right)$, follows the distribution $g\left(\alpha\left(t_{2}\right)-\alpha\left(t_{1}\right), \beta\right)$ for all $t_{1}$ $\geq 0$

- The increments of $X(t)$ are independent.

From empirical studies, the mean value and the variance of the total deterioration at time $t$ are usually expressed by means of a power law. Since the degradation of resistance of concrete structures is mainly caused by the corrosion of reinforcement, according to Ellingwood [9] a linear function, $\alpha(t)=\alpha t$, is suitable to describe the degradation of concrete structures due to corrosion of reinforcement. Consequently, the mean value and the variance of the deterioration are linear as well.

$$
E(X(t))=\frac{\alpha t}{\beta} \quad, \quad \operatorname{Var}(X(t))=\frac{\alpha t}{\beta^{2}}
$$

\section{The stochastic process of external loads}

When defining traffic loads, it is not only necessary to define the arrival distribution of traffic loads, but also to define the distribution of the loads' magnitude. For the occurrence of traffic loads, it is reasonable to adopt the Poisson process, 


$$
P(N(t)=n)=\frac{(\lambda t)^{n}}{n !} e^{-\lambda t} \quad(n=0,1,2 \ldots)
$$

for all $t \geq 0$, where $\lambda$ is the intensity of load occurrence, and $n$ is the total number of the loads arrived on the bridge in the time interval $(0, t]$.

According to the real traffic volume survey results, the axle-load distribution of different grades or variant types of roads is different. Since the axle-load distribution of heavy trucks and cars are respectively within a certain range, the distribution usually has two peaks. Hence, it is more accurate to use a superposition of several distributions to represent the axle-load distribution [10]. However, in order to simplify the problem, it is assumed that only the loads above a certain value can affect the girder. For highway bridge, this certain value refers to the superposition of girder's self-weight and light loads, so the value of the load can be expressed as a minimum load $l_{0}$ value plus a portion exceeding this minimum value. The exceeded portion $Y$ is assumed to have generalized Pareto distribution. Therefore, the corresponding maximum moment in the girder also has generalized Pareto distribution. The probability density function and cumulative distribution function of generalized Pareto distribution are given by

$$
\begin{gathered}
f_{Y}(y \mid a, c)= \begin{cases}\frac{1}{a}\left[1-\frac{c y}{a}\right]^{\left(\frac{1}{c}\right)-1} & c \neq 0, a>0 \\
\frac{1}{a} \exp \left(-\frac{y}{a}\right) & c=0, a>0\end{cases} \\
F_{Y}(y \mid a, c)= \begin{cases}1-\left[1-\frac{c y}{a}\right]^{\left(\frac{1}{c}\right)} & c \neq 0, a>0 \\
\exp \left(-\frac{y}{a}\right) & c=0, a>0\end{cases}
\end{gathered}
$$

where $a$ is scale parameter, $c$ is shape parameter.

\section{Combination of structural deterioration and loads}

For a bridge structure, if $r_{0}$ is the initial flexural capacity of a girder, and the girder is subjected to $n$ heavy loads until the age $t$ since the bridge opening, the probability of no failure during this time is the probability that the girder does not fail each time when a heavy load hits on the girder, which is the probability that all heavy loads are lower than the residual flexural capacity. According to the derivation given by Karlin [11] and Ellingwood [9], the conditional occurrence of each load has uniform distribution with density $1 / t$ in the time interval $(0, t]$. Therefore, the conditional probability that the girder will not fail is equal to the probability that the load will occur multiplied by the probability that the girder will not fail under the load, integrated from 0 to $t$.

$$
P\{\text { no failure under one load } \mid N(t)=n\}=\int_{0}^{t} \frac{1}{t} F_{Y}\left(r_{0}-l_{0}-X(\tau)\right) d \tau
$$

Since all $n$ loads occur independently with identical distribution, the survival function of the girder at age $t, S(t)$, can be obtained by the law of total probability [5], 


$$
\begin{gathered}
S(t)=P(\text { no failure in }(0, t])=E\left(\sum_{n=0}^{\infty}\left(\int_{0}^{t} \frac{1}{t} F_{Y}\left(r_{0}-l_{0}-X(\tau)\right) d \tau\right)^{n} \cdot \frac{(\lambda t)^{n}}{n !} e^{-\lambda t}\right) \\
=E\left(\exp \left(-\lambda \int_{0}^{t} 1-F_{Y}\left(r_{0}-l_{0}-X(\tau)\right) d \tau\right)\right)
\end{gathered}
$$

\section{Bayesian updating and residual life}

According to Bayes' theorem, if the prior distribution is assumed, the posterior distribution is conditional given the inspection data set $\boldsymbol{x}$ [12]. However, directly updating Eq. (7) is considerably challenging, as many parameters with unknown distributions are involved. Since the structural lifetime is the combination of deteriorating process and traffic load, and the traffic load data can be found from annual traffic survey, the only part which needs updating is the deteriorating process, which is the gamma process. Therefore, if the prior distribution of the parameters $(\alpha$ and $\beta)$ is denoted as $\pi(\alpha, \beta)$, the posterior distribution $\pi(\alpha, \beta \mid x)$ will be [13]:

$$
\pi(\alpha, \beta \mid x)=\frac{l(x \mid \alpha, \beta) \cdot \pi(\alpha, \beta)}{\iint l(\boldsymbol{x} \mid \alpha, \beta) \pi(\alpha, \beta) d \alpha d \beta} \propto l(x \mid \alpha, \beta) \cdot \pi(\alpha, \beta)
$$

where $l(\boldsymbol{x} \mid \alpha, \beta)$ is the likelihood function of the inspection data given $\alpha$ and $\beta$. Since the denominator is a constant value, the posterior $\pi(\alpha, \beta \mid x)$ is proportional to the production of the likelihood function and the prior density.

In this paper, the prior joint distribution $\pi(\alpha, \beta)$ follows the bivariate normal distribution with independent $\alpha$ and $\beta$. In reality, due to the fact that the error of measurements is always known and controllable, the variances of $\alpha$ and $\beta$ can be regarded as constant values. Thus,

$$
\pi(\alpha, \beta)=\frac{1}{2 \pi \sigma_{\alpha} \sigma_{\beta}} \exp \left(-\frac{1}{2}\left(\frac{\left(\alpha-\mu_{\alpha}\right)^{2}}{\sigma_{\alpha}^{2}}+\frac{\left(\beta-\mu_{\beta}\right)^{2}}{\sigma_{\beta}^{2}}\right)\right)
$$

For each inspection, if only one pair of parameters can be obtained (denoted as $\alpha_{i}, \beta_{i}$ ), by inference, the posterior joint probability density is conjugate to the prior joint probability density, and the updated parameters, $\alpha^{*}$ and $\beta^{*}$, happen to be the averages of the prior values and the inspected values,

$$
\pi\left(\alpha, \beta \mid \alpha_{i}, \beta_{i}\right) \propto \exp \left(-\frac{1}{2}\left(\frac{\left(\alpha-\mu_{\alpha}^{*}\right)^{2}}{\sigma_{\alpha}^{2}}+\frac{\left(\beta-\mu_{\beta}^{*}\right)^{2}}{\sigma_{\beta}^{2}}\right)\right)
$$

where

$$
\mu_{\alpha}^{*}=\frac{\mu_{\alpha}+\alpha_{i}}{2} \quad \mu_{\beta}^{*}=\frac{\mu_{\beta}+\beta_{i}}{2}
$$

Therefore, if an inspection is performed at time $T$, the survival probability distribution $S^{*}(t)$ will be calculated using the observed resistance $r_{1}$ and the new gamma process $X^{*}(t)$ with the updated parameters,

$$
S^{*}(t)=E\left(\exp \left(-\lambda \int_{0}^{t} 1-F_{Y}\left(r_{1}-l_{0}-X^{*}(\tau)\right) d \tau\right)\right)=1-F^{*}(\mathrm{t}) \quad(t>0)
$$


The survival density of the structure is obtained as the derivative of $F^{*}(t)$ :

$$
f^{*}(t)=E\left(\left(\lambda\left(1-F_{Y}\left(r_{1}-l_{0}-X^{*}(t)\right)\right)\right) \cdot \exp \left(-\lambda \int_{0}^{t} 1-F_{Y}\left(r_{1}-l_{0}-X^{*}(\tau)\right) d \tau\right)\right)
$$

Furthermore, the total life of the girder is the addition of time $T$ and the mean residual life after the inspection,

$$
M(T)=T+\int_{0}^{\infty} t \cdot f^{*}(t) d t
$$

\section{Example: A concrete highway bridge}

To illustrate how this methodology works, we consider an example of a concrete highway bridge with T cross-section girders, as this type of cross-section is relatively simple for design and construction. It is a simply supported girder bridge which consists of five girders, and all of the girders have the same cross-section. The computed span of each girder is 12 meters. The cross sections of the bridge and the girder are shown in Fig.1, and the material characteristics are given in Table 1. All girders are subjected to bending and shearing force when traffic loads exert on them, and the maximum flexural moment happens at the middle of each girder. If only the flexural capacity of the girder is taken as the main study object, the girder will fail when the bending moment caused by the vehicle load at a time is greater than the flexural capacity of the girder at that time.

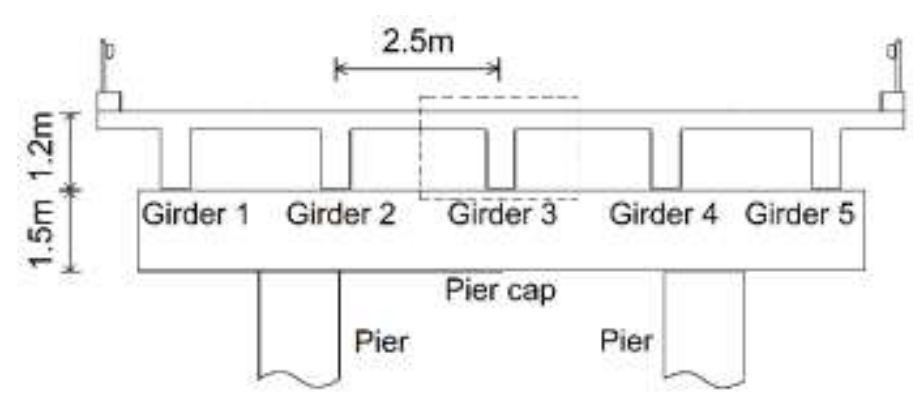

(a) Cross section of the bridge

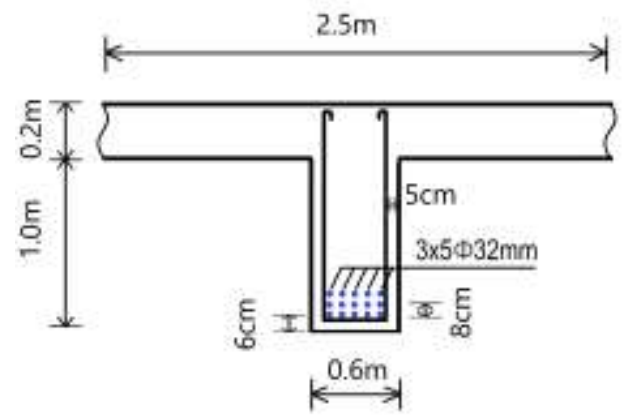

(b) Cross section of the concrete girder

Fig. 1: Cross-section details of the highway bridge.

Table 1: Some material characteristics for the concrete girders.

\begin{tabular}{|c|c|}
\hline Variable & Mean \\
\hline Steel reinforcement yield strength, $f_{y}$ & $330 \mathrm{MPa}$ \\
\hline Concrete compressive strength, $f_{c}^{\prime}$ & $22.4 \mathrm{MPa}$ \\
\hline Initial diameter of flexural reinforcement, $d_{f}$ & $31 \mathrm{~mm}$ \\
\hline The effective depth of flexural reinforcement, $h_{0}$ & $106 \mathrm{~cm}$ \\
\hline
\end{tabular}

When the bridge construction is completed and the bridge starts operation, the initial flexural capacity of each girder $\left(M_{c p}\right)$ is $4713.25 \mathrm{kN} \cdot \mathrm{m}$ [14]. The density of RC is about $2500 \mathrm{~kg} / \mathrm{m}^{3}$. Except for the bending moment generated by the self-weight, $485.1 \mathrm{kN} \cdot \mathrm{m}$, the flexural capacity of each main beam is $4228.15 \mathrm{kN} \cdot \mathrm{m}$. If the bending moment generated by trucks with the gross weight less than or equal to 17.5 tons (i.e., less than $514.5 \mathrm{kN} \cdot \mathrm{m}$ ) is negligible, according to the axle-load spectrum obtained from the traffic volume survey, the portion of the bending moment exceeding $514.5 \mathrm{kN} \cdot \mathrm{m}$ caused by traffic loads can be fitted by the generalized Pareto distribution with $a=0.5$ and $c=0.02$. The bending moment exceeding $514.5 \mathrm{kN} \cdot \mathrm{m}$ caused by traffic loads occurs about 4000 times per year. 
By investigating similar bridges under the same environmental conditions, bridge engineers estimate that the mean value of the deterioration rate of flexural capacity is $9.78 \mathrm{kN} \cdot \mathrm{m} / \mathrm{year}$, and the corresponding variation is 6.5 . Hence, the parameters of the gamma distribution are $\alpha=14.715$ and $\beta=1.505$. The prior joint normal distribution of the parameters of the gamma distribution is shown in Table2. Based on Eq. (7), the prior probability density function of the lifetime can be calculated by Monte Carlo method.

When the bridge has been in operation for 10 years, by measuring the diameter of steel bars of the five girders, the bridge engineers found that the flexural moment capacity is $4550 \mathrm{kN} \cdot \mathrm{m}$, and the deterioration rate of flexural moment capacity is $16.84 \mathrm{kN} \cdot \mathrm{m} /$ year, with the corresponding variation of 8.8 . Consequently, the corresponding $\alpha$ and $\beta$ values are equal to 32.226 and 1.914, respectively. In addition, the new traffic survey shows that the bending moment exceeding 514.5 $\mathrm{kN} \cdot \mathrm{m}$ caused by traffic loads occurs 5000 times per year. By applying Bayesian updating, the posterior distribution can be computed (see Table 2), and the corresponding posterior distribution of lifetime can also be computed by Monte Carlo method.

Table 2: Comparison of prior and posterior parameters.

\begin{tabular}{|c|c|c|c|c|}
\hline Parameter & $\begin{array}{c}\text { Initial } \\
\text { Mean }(\mu)\end{array}$ & $\begin{array}{c}\text { Updated } \\
\text { Mean }\left(\mu^{\prime}\right)\end{array}$ & $\begin{array}{c}\text { Variance } \\
\left(\sigma^{2}\right)\end{array}$ & $\begin{array}{c}\text { 95\% CI of Updated } \\
\text { Mean }\end{array}$ \\
\hline$\alpha$ & 14.715 & 23.47 & 0.5 & $(22.65,24.29)$ \\
\hline$\beta$ & 1.505 & 1.710 & 0.08 & $(1.5788,1.8412)$ \\
\hline
\end{tabular}

Fig.2 shows the comparison of prior and posterior cumulative distribution functions and the probability density functions of the residual life of girder after the inspection in the $10^{\text {th }}$ year. The structure was found to degrade significantly faster than the initial expectation, which is most likely due to the increase in traffic volume and the increase in heavy vehicles within the ten years. This scenario is very common in most highway bridges. In the actual operation of the bridge, once this situation happens, the previous maintenance plan should be revised in time to ensure the normal operation of the bridge under the new degrading law.

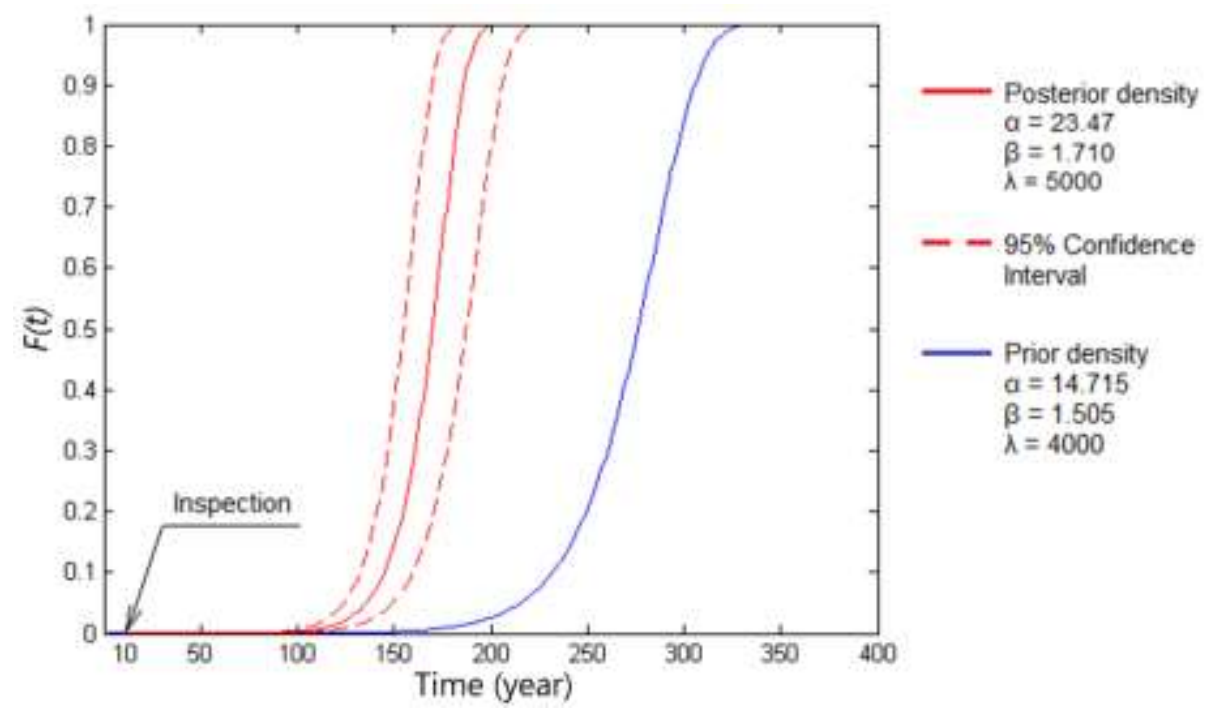

(a) Cumulative distribution function of the girder's residual life 


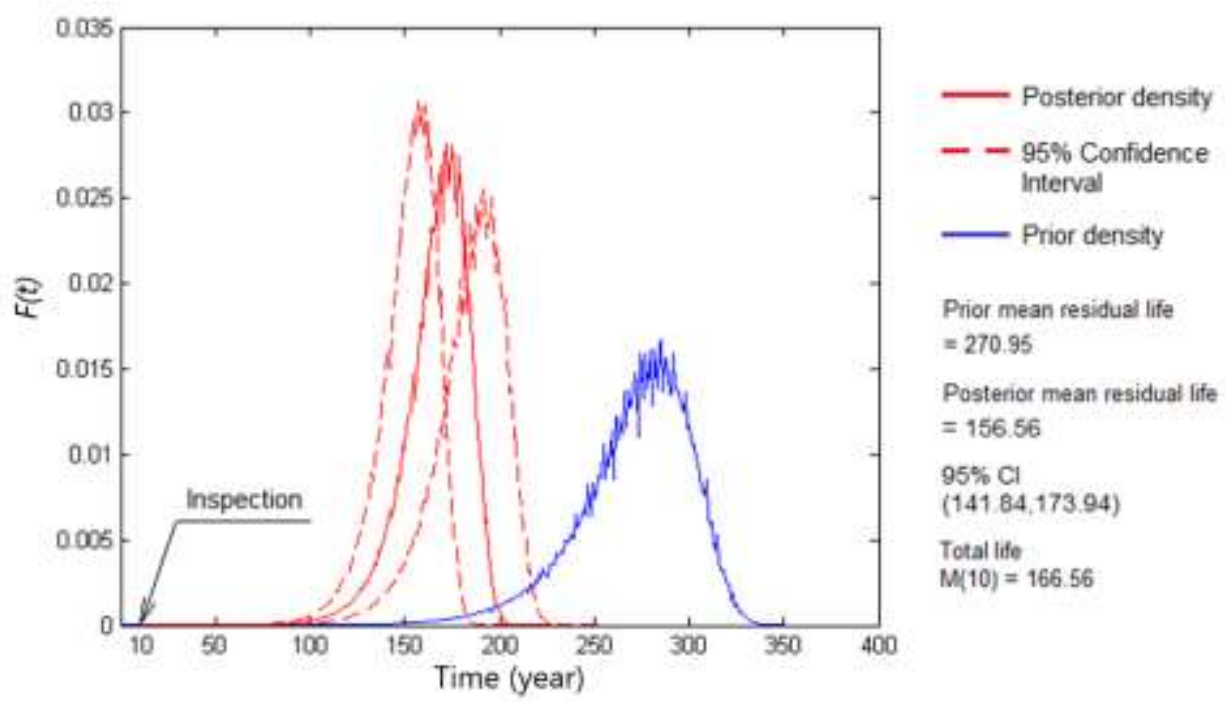

(b) Probability density function of the girder's residual life

Fig. 2: Comparison of the prior and the posterior distribution of the girder's lifetime

\section{Conclusions}

This paper combines the vehicle loads acting on the bridge with the deterioration of concrete and updates the residual life of the bridge structure by the Bayesian updating method. Since directly updating the structural lifetime distribution is complicated, the deteriorating process of concrete modeled as the gamma process and the effect of vehicle loads modeled as the Poisson process and generalized Pareto distributed random variable are considered. After each inspection, the gamma process is updated by Bayesian method. The effect of traffic loads can be obtained from the latest report from government or related institutions. Finally, the cumulative distribution function and probability density function of structural residual life can be calculated by Monte Carlo method. This method can effectively take advantage of the inspection data to guide the future maintenance of infrastructure.

\section{Acknowledgements}

The authors would like to thank the Natural Sciences and Engineering Research Council of Canada for the financial support under Grant No. RGPIN 121384-11.

\section{References}

[1] M. P. Enright and D. M. Frangopol, "Condition prediction of deteriorating concrete bridges using Bayesian updating," Journal of Bridge Engineering, vol. 125, pp. 1118-1125, 1999.

[2] Q. Suo and M. G. Stewart, "Corrosion cracking prediction updating of deteriorating RC structures using inspection information," Reliability Engineering \& System Safety, vol. 94, pp. 1340-1348, 2009.

[3] S. A. Miran, Q. Huang, and H. Castaneda, "Time-Dependent Reliability Analysis of Corroded Buried Pipelines Considering External Defects," Journal of Infrastructure Systems, vol. 22, pp. 04016019, 2016.

[4] F. Hong and J. A. Prozzi, "Estimation of pavement performance deterioration using Bayesian approach," Journal of Infrastructure Systems, vol. 12, pp. 77-86, 2006.

[5] J. M. van Noortwijk, J. A. M. van der Weide, M. J. Kallen, and M. D. Pandey, "Gamma processes and peaks-overthreshold distributions for time-dependent reliability," Reliability Engineering \& System Safety, vol. 92, pp. 1651-1658, 2007.

[6] K. V. Yuen, "Recent developments of Bayesian model class selection and applications in civil engineering," Structural Safety, vol. 32, pp. 338-346, 2010. 
[7] J. L. Beck and S.-K. Au, "Bayesian updating of structural models and reliability using Markov chain Monte Carlo simulation," Journal of Engineering Mechanics, vol. 128, pp. 380-391, 2002.

[8] J. M. van Noortwijk, "A survey of the application of gamma processes in maintenance," Reliability Engineering \& System Safety, vol. 94, pp. 2-21, 2009.

[9] B. R. Ellingwood and Y. Mori, "Probabilistic methods for condition assessment and life prediction of concrete structures in nuclear power plants," Nuclear Engineering Design, vol. 142, pp. 155-166, 1993.

[10] L. F. Macea, L. Márquez, and H. LLinás, "Improvement of axle load spectra characterization by a mixture of three distributions," Journal of Transportation Engineering, vol. 141, 2015.

[11] S. Karlin, A first course in stochastic processes. Academic Press, 2014.

[12] R. W. HF Martz, Bayesian Reliability Analysis. New York: Wiley, 1982.

[13] B. Guo and L. Tan, "Reliability assessment of gamma deteriorating system based on Bayesian updating," in 8th International Conference on Reliability, Maintainability and Safety, 2009, pp. 429-432.

[14] W. F. Chen and L. Duan, Handbook of international bridge engineering. CRC Press, 2013. 